\title{
MANOBRAS DE ATITUDE DE SATÉLITES ARTIFICIAIS COM ÂNGULOS DE EULER
}

\author{
MARIA CECÍLIA ZANARDI ${ }^{1}$, JOÃO VITOR LEMOS FRANÇA ${ }^{2}$ \\ 1,2. Faculdade de Engenharia - UNESP - Campus de Guaratinguetá \\ Av. Dr. Ariberto Pereira da Cunha, 333, 12513-060, Guaratinguetá, Brasil \\ E-mail: cecília@feg.unesp.br, joao.v.l.franca@gmail.com
}

\begin{abstract}
Artificial satellites attitude maneuvers simulations are made using the MATLAB software, such as the magnitude of the propulsive control torques are enough to change of the attitude parameter satellite of the initial values to the previous stabilized values. The temporal behavior between the propulsive torque and the components of spin velocity and Euler angles are analyzed during the maneuver.
\end{abstract}

Keywords_ Engineer applications, attitude maneuvers, Euler equations, kinematics equations.

Resumo-Simulações de manobras de atitude de satélites artificiais são realizadas com o software MATLAB, de modo que as magnitudes dos torques propulsivos de controle utilizados sejam suficientes e necessárias para a mudança dos parâmetros da atitude do satélite em questão de valores iniciais a valores finais previamente estabelecidos. São analisados os comportamentos temporais entre as componentes do torque propulsivo e as componentes da velocidade de rotação e os ângulos de Euler, bem como a conjunção do comportamento de todas estas variáveis na determinação da própria manobra.

Palavras-chave— Aplicações de engenharia, manobras de atitude, equações de Euler, equações cinemáticas.

\section{Introdução}

O movimento de um satélite artificial em torno da Terra é caracterizado pelo seu movimento translacional e rotacional, sendo o enfoque deste trabalho o movimento rotacional. Este movimento é caracterizado pela orientação do satélite no espaço e pela sua velocidade de rotação. A orientação espacial do satélite é denominada de atitude e sua análise é muito importante, pois da orientação e posicionamento precisos do satélite dependerá o sucesso da missão a que ele se destina.

Imagine um satélite em órbita terrestre que deva apontar suas antenas sempre em direção à Terra. Ao longo do tempo as perturbações ambientais ocasionam mudanças na orientação espacial (atitude) do satélite, fazendo com que suas antenas não apontem mais em direção à Terra e tornando assim a comunicação inviável, o que prejudica a missão do satélite. Para que isso não ocorra são necessárias algumas manobras de atitude ao longo da vida útil do satélite.

O objetivo deste trabalho é analisar os resultados das simulações de manobras de atitude de satélites artificiais utilizando torques propulsivos de pequena magnitude. As simulações numéricas são realizadas com o software MATLAB. Neste trabalho a atitude do satélite é descrita pelos ângulos de Euler, e as equações do movimento são dadas pelas equações dinâmicas de Euler e equações cinemáticas. Nas equações dinâmicas do movimento rotacional são introduzidos torques de controle, de modo a auxiliar na mudança de uma orientação inicial do satélite para uma orientação desejada, ou seja, através dos torques propulsivos de controle são realizadas manobras de atitude, as quais serão de pequena magnitude com a orientação desejada sendo próxima da inicial.

Os resultados das simulações mostram o comportamento temporal dos componentes da velocidade de rotação e dos ângulos de Euler durante o tempo de duração da manobra. Tais resultados são úteis para se observar as características dos componentes do torque de propulsão e qual a magnitude necessária para a realização de uma manobra de atitude, de modo a manter a atitude do satélite dentro da precisão requerida na missão espacial a que ele se destina.

\section{Equações do Movimento}

As equações do movimento rotacional são dadas pelas equações dinâmicas e cinemáticas apresentadas a seguir.

\subsection{Equações Dinâmicas}

As equações dinâmicas do movimento relacionam as taxas de variação das componentes $p, q$ e $r$ da velocidade de rotação $W$ com as componentes $N_{x}, N_{y}$ e $N_{z}$ do torque externo atuante no sistema, sendo dadas por (Wertz, 1978;Zanardi,2005):

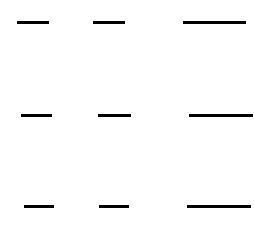

onde $I_{x}, I_{y}$ e $I_{z}$ são os momentos principais de inércia do satélite; $p, q$ e $r$ são as componentes da velocidade de rotação no sistema do satélite (com eixos paralelos aos eixos principais de inércia do satélite); $N_{x}, N_{y}$ e $N_{z}$ são componentes dos torques externos no sistema do satélite, neste caso apenas o torque propulsivo. 


\subsection{Equações Cinemáticas}

A orientação espacial do satélite é aqui descrita pelos ângulos de Euler $\phi, \theta, \psi$, da sequência 3-1-3 (Pisacane E Moore, 1994; Zanardi, 2009), representados na Figura 1.

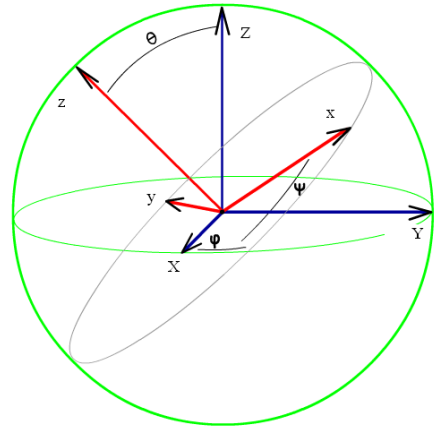

Figura 1 - Sequência 3-1-3 dos ângulos de Euler $\phi, \theta, \psi$, OXYZ - sistema inercial e Oxyz - sistema fixo no satélite.

As equações cinemáticas descrevem as taxas de variação temporal dos ângulos de Euler em função das componentes da velocidade de rotação $p, q$ e $r$ no sistema fixo no satélite, sendo dadas por (Pisacane E Moore, 1994; Zanardi, 2009):

\section{Resultados}

As manobras de atitude são realizadas através da integração numérica das equações $1-6$, incluindo apenas o torque propulsivo nas equações dinâmicas, de modo a levar a atitude de um valor inicial ao valor requerido previamente estabelecido, dentro das precisões desejadas. As integrações são realizadas com o auxilio do método de Kunge Kutta de $4^{\mathrm{a}}$ ordem e do sofatware MATLAB.

As aplicações são realizadas para os dados disponíveis para o Segundo Satélite Brasileiro de Coleta de Dados SCD2, disponibilizadas pelo Centro de Controle de Satélite CCS do INPE. Destaque-se que o SCD2 possui controle de atitude através de rodas de reação e não com atuação de torque propulsivo como é aqui realizado. Para este satélite a velocidade de rotação alinha-se ao longo do eixo de maior momento principal de inércia, de modo que o módulo da velocidade de rotação $W$ deve ser a sua componente $r$. Dentre os dados disponíveis foram escolhidos 5 instantes para a realização das manobras, sendo que a Tabela 1apresenta os dados do CCS/INPE para tais instantes e são coincidentes com a atuação do controle pelo CCS realizadas na época.

Os ângulos de atitude correspondem aos ângulos de Euler $\varphi$ e $\theta$, associados aos ângulo de posicionamento do eixo de rotação ( ascensão reta e declinação). Nas simulações também é analisada a magnitude da velocidade de rotação W.
Na Tabela 2 é apresentado o número total de simulações realizadas para cada manobra (França, 2013), quantificando o número de manobras que foram adequadas de acordo com a precisão requerida pelo CCS/INPE, devendo ser de $0,5^{\circ}$ para ângulos e de $0,0524 \mathrm{rad} / \mathrm{s}(0,5 \mathrm{rpm})$ para a velocidade de rotação. Para cada manobra foram realizadas diversas simulações utilizando diferentes valores para as magnitudes dos torques de controle, totalizando 248 simulações para as 5 manobras previstas.

Detalhes de resultados obtidos nas simulações foram selecionados e estão apresentados a seguir, destacando as simulações que estão dentro da precisão requerida da missão.

Tabela 1 - Dados de atitude para o SCD2: valor inicial e requerido nas manobras.

\begin{tabular}{|c|c|c|c|c|}
\hline Manobra & Dia & $\boldsymbol{\varphi}\left(^{\mathbf{o}}\right)$ & $\boldsymbol{\theta}\left(^{\mathbf{o}}\right)$ & $\mathbf{W}(\mathbf{r a d} / \mathbf{s})$ \\
\hline \multirow{2}{*}{1} & $\begin{array}{c}\text { Início da Manobra: } \\
11 / 02 / 2002\end{array}$ & 10.31 & 26.2137 & 3.6233 \\
\cline { 2 - 5 } & Valor requerido & 8.71 & 26.53 & 3.6107 \\
\hline \multirow{2}{*}{2} & $\begin{array}{c}\text { Início da Manobra: } \\
23 / 02 / 2002\end{array}$ & 8.23 & 26.641 & 3.5427 \\
\cline { 2 - 5 } & Valor requerido & 6.6 & 28.78 & 3.528 \\
\hline \multirow{3}{*}{3} & $\begin{array}{c}\text { Início da Manobra: } \\
\text { 28/02/2002 }\end{array}$ & 5.64 & 29.5322 & 3.4976 \\
\cline { 2 - 5 } & Valor requerido & 3.75 & 30.5998 & 3.5008 \\
\hline \multirow{3}{*}{4} & $\begin{array}{c}\text { Início da Manobra: } \\
\text { 04/03/2002 }\end{array}$ & 2.52 & 31.427 & 3.4924 \\
\cline { 2 - 5 } & Valor requerido & 1.63 & 31.75 & 3.4914 \\
\hline
\end{tabular}

Tabela 2. Quadro geral das manobras e simulações de atitude realizadas: $\mathbf{A Q}$ - manobras adequadas, $\mathbf{A Q W} \boldsymbol{\varphi}$ - manobras adequadas para $W$ e $\varphi$, AQW $\theta$ - manobras adequadas para $W$ e $\theta, \mathbf{I A Q}$ - manobras inadequedas, TS - total de simulações.

\begin{tabular}{|c|c|c|c|c|c|}
\hline Manobra & AQ & AQW $\boldsymbol{~}$ & AQW $\boldsymbol{\theta}$ & IAQ & TS \\
\hline 1 & 0 & 1 & 9 & 27 & 37 \\
\hline 2 & 0 & 6 & 2 & 64 & 72 \\
\hline 3 & 3 & 2 & 0 & 54 & 59 \\
\hline 4 & 2 & 1 & 0 & 32 & 35 \\
\hline
\end{tabular}

\section{Discussões}

Como se observa na Tabela 2 , apenas as manobras 3 e 4 atingiram os valores desejados dentro da precisão requerida, sendo que a manobra 1 só satisfez os requisitos para o ângulo $\theta$ e velocidade de rotação $W$, enquanto que as manobras 2 e 3 satisfizeram os requisitos para $\mathrm{W}$ e isoladamente os ângulos $\theta$ ou $\varphi$.

Para a manobra 3, realizada para os dados iniciais do dia 28/02/2002, dentre as 59 simulações realizadas para diferentes valores de torque de propulsão, 3 se enquadram dentro da precisão requerida. Na Tabela 3 se encontram detalhes das 
simulações destacando o tempo de duração (TDM) da manobra, os valores das componentes $N_{x}, N_{y}, N_{z}$ dos torques de propulsão no sistema do satélite, valores após manobra obtidos para os ângulos $\varphi, \theta$ e componentes da velocidade de rotação $p, q, r$ no sistema do satélite e suas respectivas diferenças $\Delta \varphi, \Delta \theta, \Delta p, \Delta q, \Delta r$ em relação os valores requeridos. Observa-se por estes resultados, que as precisões só foram alcançadas devido a aplicação de um grande torque propulsivo, em especial com grande componente $N_{\mathrm{y}}$, o que na prática não é viável para uma manobra de atitude. Pelas equações cinemáticas 4 e 5 verificam-se as variações de $\varphi, \theta$ são afetadas pelas componentes $p$ e $q$ da velocidade de rotação. Pelas equações cinemáticas e pelas condições iniciais justifica-se a necessidade de grande valor para a componente $N_{y}$ do torque propulsivo.

Tabela 3 - Resultados das simulações da Manobra 3

\begin{tabular}{|c|c|c|c|}
\hline $\begin{array}{c}\text { Manobra } \\
\mathbf{3}\end{array}$ & $\begin{array}{c}\text { Simulação } \\
\mathbf{1}\end{array}$ & $\begin{array}{c}\text { Simulação } \\
\mathbf{2}\end{array}$ & $\begin{array}{c}\text { Simulação } \\
\mathbf{3}\end{array}$ \\
\hline$T D M(\mathrm{~s})$ & 40 & 30 & 20 \\
\hline$N_{x}(\mathrm{Nm})$ & -1 & 2 & 3 \\
\hline$N_{y}(\mathrm{Nm})$ & 20 & -30 & -40 \\
\hline$N_{z}(\mathrm{Nm})$ & 1,43 & 1,7 & 1,73 \\
\hline$\varphi\left(^{\circ}\right)$ & 3,7476 & 3,9511 & 3,7182 \\
\hline$\theta\left(^{\circ}\right)$ & 30,3950 & 30,644 & 30,4497 \\
\hline$p(\mathrm{rad} / \mathrm{s})$ & 0,017 & 0,0171 & 0,0173 \\
\hline$q(\mathrm{rad} / \mathrm{s})$ & $-0,079$ & 0,071 & $-0,907$ \\
\hline$r(\mathrm{rad} / \mathrm{s})$ & 3,5025 & 3,5008 & 3,5001 \\
\hline$\Delta \varphi\left(^{\circ}\right)$ & 0,0024 & 0,2011 & 0,015 \\
\hline$\Delta \theta\left(^{\circ}\right)$ & 0,1048 & 0,0442 & 0,0284 \\
\hline$\Delta p(\mathrm{rad} / \mathrm{s})$ & 0,017 & 0,0171 & 0,0263 \\
\hline$\Delta q(\mathrm{rad} / \mathrm{s})$ & 0,079 & 0,071 & 0,023 \\
\hline$\Delta r(\mathrm{rad} / \mathrm{s})$ & 0,017 & 0 & 0,0003 \\
\hline
\end{tabular}

Para a manobra 5, realizada para os dados iniciais do dia 04/03/2002, dentre as 35 simulações realizadas para diferentes valores de torque de propulsão, 2 se enquadram dentro da precisão requerida, cujos detalhes são apresentados na Tabela 4. Nesta manobra a magnitude do torque de propulsão foi menor do que na manobra 4, mas mesmo assim além dos valores possíveis para uma aplicação prática para manutenção da atitude requerida do satélite.
Tabela 4 - Resultados das simulações da Manobra 5

\begin{tabular}{|c|c|c|}
\hline $\begin{array}{c}\text { Manobra } \\
4\end{array}$ & $\underset{1}{\text { Simulação }}$ & $\begin{array}{c}\text { Simulação } \\
2\end{array}$ \\
\hline$T D M(s)$ & 35 & 45 \\
\hline$N_{x}(\mathrm{Nm})$ & $-2,5$ & 4 \\
\hline$N_{y}(\mathrm{Nm})$ & 13 & -15.23 \\
\hline$N_{z}(N m)$ & $-0,7$ & 0,738 \\
\hline$\varphi\left(^{o}\right)$ & 1,79 & 1,645 \\
\hline$\theta\left(^{o}\right)$ & 31,73 & 31,7784 \\
\hline$p(\mathrm{rad} / \mathrm{s})$ & $-0,21$ & 0,0263 \\
\hline$q(\mathrm{rad} / \mathrm{s})$ & 0,079 & 0,023 \\
\hline$r(\mathrm{rad} / \mathrm{s})$ & 3,4916 & 3,4911 \\
\hline$\Delta \varphi\left(^{o}\right)$ & 0,16 & 0,015 \\
\hline$\Delta \theta\left(^{o}\right)$ & 0,02 & 0,0284 \\
\hline$\Delta p(\mathrm{rad} / \mathrm{s})$ & $-0,021$ & 0,0263 \\
\hline$\Delta q(\mathrm{rad} / \mathrm{s})$ & 0,079 & 0,023 \\
\hline$\Delta r(\mathrm{rad} / \mathrm{s})$ & 0,0002 & 0,0003 \\
\hline
\end{tabular}

As manobras 1 e 2 não conseguiram atingir simultaneamente os valores requeridos para os dois ângulos $\theta$ e $\varphi$. Os resultados de 2 simulações de cada manobra estão apresentados nas Tabelas 5 e 6 . Nota-se que os valores das componentes do torque de propulsão são adequados para uma aplicação prática, quando apenas o valor requerido de $\theta$ é satisfeito juntamente com a velocidade de rotação. Observa-se novamente um grande valor da componente $N_{y}$ do torque de propulsão para satisfazer os valores requeridos para o ângulo $\varphi$.

Nas 37 simulações realizadas para a manobra 1 apenas um obteve o valor requerido para o ângulo $\varphi$, enquanto 9 atingiram o valor desejado para o ângulo $\theta$.

Nas 72 simulações realizadas para a manobra 2 apenas duas obtiveram o valor requerido para o ângulo $\theta$, enquanto 6 atingiram o valor desejado para $\varphi$. 
Tabela 5 - Resultados das simulações da Manobra 1

\begin{tabular}{|c|c|c|}
\hline Manobra & Simulação & Simulação \\
$\mathbf{1}$ & & $\mathbf{2}$ \\
\hline$T D M(s)$ & 30 & 30 \\
\hline$N_{x}(\mathrm{Nm})$ & 0,1 & 0,1 \\
\hline$N_{y}(\mathrm{Nm})$ & $-16,375$ & -5 \\
\hline$N_{z}(\mathrm{Nm})$ & $-7,55$ & $-7,55$ \\
\hline$\Delta \varphi\left(^{\circ}\right)$ & 0,5775 & 1,501 \\
\hline$\Delta \theta\left(^{\circ}\right)$ & 0,0284 & 0,0002 \\
\hline$\Delta p(\mathrm{rad} / \mathrm{s})$ & 0,066 & 0,0118 \\
\hline$\Delta q(\mathrm{rad} / \mathrm{s})$ & 0,0392 & 0,0025 \\
\hline$\Delta r(\mathrm{rad} / \mathrm{s})$ & 0,0000 & 0,0000 \\
\hline
\end{tabular}

Tabela 6 - Resultados das simulações da Manobra 2

\begin{tabular}{|c|c|c|}
\hline Manobra & Simulação & Simulação \\
$\mathbf{2}$ & $\mathbf{2}$ & \\
\hline$T D M(s)$ & 50 & 40 \\
\hline$N_{x}(\mathrm{Nm})$ & -1 & 0,2 \\
\hline$N_{y}(\mathrm{Nm})$ & -15 & 0,2 \\
\hline$N_{z}(\mathrm{Nm})$ & $-4,3$ & 0,3 \\
\hline$\Delta \varphi\left(^{\circ}\right)$ & 0,012 & 1,169 \\
\hline$\Delta \theta\left(^{\circ}\right)$ & 0,9753 & 0,0284 \\
\hline$\Delta p(\mathrm{rad} / \mathrm{s})$ & 0,0059 & 0,0263 \\
\hline$\Delta q(\mathrm{rad} / \mathrm{s})$ & 0,0606 & 0,0023 \\
\hline$\Delta r(\mathrm{rad} / \mathrm{s})$ & 0,0041 & 0,0833 \\
\hline
\end{tabular}

\section{Comentários Finais}

Neste trabalho foram realizadas 4 diferentes manobras de atitude com o objetivo de se analisar a magnitude de torque de propulsão necessária para se passar de um valor inicial da atitude para um valor requerido. Em cada manobra foram realizadas diversas simulações, considerando diferentes valores para o torque de propulsão. A atitude do satélite foi representada por ângulos de Euler e as equações dinâmicas de Euler utilizadas para introduzir a atuação do torque propulsivo.

Aplicações foram realizadas com os dados do SCD2. Verificou-se que o valor requerido para o ângulo $\varphi$ só é possível para altos valores do torque de propulsão, os quais são inviáveis na prática. Isso ocorre pois nas manobras consideradas a mudança no ângulo $\varphi$ é da ordem de $2^{\circ}$, e porque existe uma interação grande entre as equações que descrevem a taxa de variação de $\varphi$ e de $\theta$ com as componentes da velocidade de rotação nos eixos x e $\mathrm{y}$.

Para o ângulo $\theta$ e para a velocidade de rotação $W$ foi possível obter magnitudes de torque propulsivo viáveis para uma aplicação prática. Saliente-se que as variações em $\theta$ são pequenas, menores que $1^{\circ}$ (exceção da manobra 2$)$, em relação as variações requeridas em $\varphi$, o que possibilitou atingir o valor requerido com torques propulsivos menores.

Saliente que neste trabalho nenhum sistema de controle foi introduzido, tendo sido analisada a resposta do sistema de equações do movimento à atuação do torque propulsivo, através de integração numérica pelo método de Kunge Kutta de $4^{\text {a }}$ ordem no software MATLAB. Resultados mais adequados podem ser obtido com um estudo detalhado de um sistema de controle de atitude.

\section{Agradecimentos}

Os autores agradecem o apoio do $\mathrm{CNPq}$, através do programa PIBIC-Jr, e da FAPESP através do processo no. 2012/21023-6.

\section{Referências Bibliográficas}

Fonseca, J. B. S. (2010). Manobras de órbita e atitude de satélites artificiais. Relatório Final do INPE. CNPq/PIBIC, São José dos Campos.

França, J. V. L. (2013). Manobras de attitude de Satélties Artificiais. Relatório Final de Bolsa PIBIC-Jr/CNPq. UNESP - Campus de Guaratinguetá.

Pisacane, V. L.; Moore, R. C. (1994). Fundamentals of Space Systems. Oxford University Press, New York.

Prado, A. F. B. A.; Kuga, H. K. (2001). Fundamentos de Tecnologia Espacial. INPE, São José dos Campos.

Wertz, J. R. (1978). Spacecraft Attitude Determination and Control, D. Reidel, Dordrecht, Holanda. 
Zanardi, M. C. (2005). Dinâmica da Atitude de Satélites Artificiais, Tese de Livre Docência, Faculdade de Engenharia - UNESP - Campus de Guaratinguetá, Guaratinguetá- SP.

Zanardi, M. C. (2009). Representações de Atitude, Proceeding of the 8th Brazilian Conference on Dynamics, Control and Applications, p.1 -14. 